\title{
Gaussian approximation to the extreme value index estimator of a heavy-tailed distribution under random censoring
}

\author{
Brahim Brahimi, Djamel Meraghni, Abdelhakim Necir* \\ Laboratory of Applied Mathematics, Mohamed Khider University, Biskra, Algeria
}

\begin{abstract}
We make use of the empirical process theory to approximate the adapted Hill estimator, for censored data, in terms of Gaussian processes. Then, we derive its asymptotic normality, only under the usual second-order condition of regular variation. Our methodology allows to relax the assumptions, made in Einmahl et al. (2008), on the heavy-tailed distribution functions and the sample fraction of upper order statistics.
\end{abstract}

Keywords: Empirical process; Gaussian approximation; Hill estimator; Random censoring.

AMS 2010 Subject Classification: 60G70, 60F17, 62G30.

*Corresponding author: necirabdelhakim@yahoo.fr

E-mail addresses:

brah.brahim@gmail.com (B. Brahimi)

djmeraghni@yahoo.com (D. Meraghni) 


\section{Introduction}

For $n \geq 1$, let $X_{1}, X_{2}, \ldots, X_{n}$ be $n$ independent copies of a non-negative continuous random variable $(\mathrm{rv}) X$, defined over some probability space $(\Omega, \mathcal{A}, \mathbb{P})$, with cumulative distribution function (cdf) $F$. We assume that the distribution tail $1-F$ is regularly varying at infinity, with index $\left(-1 / \gamma_{1}\right)$, notation: $1-F \in \mathcal{R} \mathcal{V}_{\left(-1 / \gamma_{1}\right)}$. That is

$$
\lim _{t \rightarrow \infty} \frac{1-F(t x)}{1-F(t)}=x^{-1 / \gamma_{1}}, \text { for any } x>0,
$$

where $\gamma_{1}>0$, called shape parameter or tail index or extreme value index (EVI), is a very crucial parameter in the analysis of extremes. It governs the thickness of the distribution right tail: the heavier the tail, the larger $\gamma_{1}$. Its estimation has got a great deal of interest for complete samples, as one might see in the textbook of Beirlant et al. (2004). In this paper, we focus on the most celebrated (consistent and asymptotically normal) estimator of $\gamma_{1}$, that was proposed by Hill (1975):

$$
\widehat{\gamma}_{1}^{H}=\widehat{\gamma}_{1}^{H}(k):=\frac{1}{k} \sum_{i=1}^{k} \log X_{n-i+1: n}-\log X_{n-k: n}
$$

where $X_{1: n} \leq \ldots \leq X_{n: n}$ are the order statistics pertaining to the sample $\left(X_{1}, \ldots, X_{n}\right)$ and $k=k_{n}$ is an integer sequence satisfying

$$
1<k<n, k \rightarrow \infty \text { and } k / n \rightarrow 0 \text { as } n \rightarrow \infty
$$

In the analysis of lifetime, reliability or insurance data, the observations are usually randomly censored. In other words, in many real situations the variable of interest $X$ is not always available. An appropriate way to model this matter, is to introduce a non-negative continuous rv $Y$, called censoring $\mathrm{rv}$, independent of $X$ and then to consider the $\operatorname{rv} Z:=\min (X, Y)$ and the indicator variable $\delta:=\mathbf{1}(X \leq Y)$, which determines whether or not $X$ has been observed. The cdf's of $Y$ and $Z$ will be denoted by $G$ and $H$ respectively. The analysis of extreme values of randomly censored data is a new research topic to which Reiss and Thomas (1997) made a very brief reference, in Section 6.1, as a first step but with no asymptotic results. Considering Hall's model, Beirlant et al. (2007) proposed estimators for the EVI and high quantiles and discussed their asymptotic properties, when the data are censored by a deterministic threshold. More recently, Einmahl et al. (2008) adapted various EVI estimators to the case where data are censored, by a random threshold, and proposed a unified method to establish their asymptotic normality. The obtained 
estimators are then used in the estimation of extreme quantiles under random censorship. Moreover, they applied their results on the Australian aids survival data available in the MASS-package of the R software. Gomes and Neves (2011) also made a contribution to this field by providing a detailed simulation study and applying the estimation procedures on some survival data sets. In the same context, Worms and Worms (2014) presented a new approach, based on Kaplan-Meier integration, to define an estimator for positive tail index and prove its consistency.

We start by a reminder of the definition of the adapted Hill estimator, of the tail index $\gamma_{1}$, under random censorship. The tail of the censoring distribution is assumed to be regularly varying too, that is $1-G \in \mathcal{R} \mathcal{V}_{\left(-1 / \gamma_{2}\right)}$, for some $\gamma_{2}>0$. By virtue of the independence of $X$ and $Y$, we have $1-H(x)=(1-F(x))(1-G(x))$ and therefore $1-H \in \mathcal{R} \mathcal{V}_{(-1 / \gamma)}$, with $\gamma:=\gamma_{1} \gamma_{2} /\left(\gamma_{1}+\gamma_{2}\right)$. Let $\left\{\left(Z_{i}, \delta_{i}\right), 1 \leq i \leq n\right\}$ be a sample from the couple of rv's $(Z, \delta)$ and $Z_{1: n} \leq \ldots \leq Z_{n: n}$ the order statistics pertaining to $\left(Z_{1}, \ldots, Z_{n}\right)$. In the sequel, the functions

$$
H^{(j)}(v):=\mathbb{P}(Z \leq v, \delta=j), j=0,1,
$$

will play a prominent role. If we denote the concomitant of the $i$ th order statistic by $\delta_{[i: n]}$ (i.e. $\delta_{[i: n]}=\delta_{j}$ if $Z_{i: n}=Z_{j}$ ), then the adapted Hill estimator of $\gamma_{1}$ is defined by $\widehat{\gamma}_{1}:=\widehat{\gamma}^{H} / \widehat{p}$, where $\widehat{\gamma}^{H}$ represents Hill's estimator of $\gamma$ and $\widehat{p}:=k^{-1} \sum_{i=1}^{k} \delta_{[n-i+1: n]}$ estimates $p:=\gamma / \gamma_{1}$. Einmahl et al. (2008) established the asymptotic normality of $\widehat{\gamma}_{1}$ by assuming that cdf's $F$ and $G$ are absolutely continuous and that the quantile function $U_{H}(t):=H^{\leftarrow}(1-1 / t), t \geq 1$, (the notation $K^{\leftarrow}$ stands for the quantile function pertaining to a cdf $K$ ) satisfies the second-order condition of regular variation (see de Haan and Stadtmüller, 1996), that we write as follows:

$$
\lim _{t \rightarrow \infty} \frac{U_{H}(t x) / U_{H}(t)-x^{\gamma}}{A^{*}(t)}=x^{\gamma} \frac{x^{\tau}-1}{\tau}, \text { for all } x>0
$$

where $\tau<0$ is the second-order parameter and $A^{*}(t)$ is a function tending to zero and not changing sign near infinity. For a discussion on the relationships between (1.3) and other representations of the second-order condition of regular variation, like that used in Einmahl et al. (2008), one refers to Fraga Alves et al. (2007). In addition, Einmahl et al. (2008) made three conditions $\left[\mathcal{H}_{1}\right]-\left[\mathcal{H}_{3}\right]$ (equivalently stated below) on the sequence $k$ in terms of $U_{H}$ and an auxiliary function

$$
\mathbf{p}(z):=\frac{\bar{G}(z) f(z)}{\bar{G}(z) f(z)+\bar{F}(z) g(z)},
$$


where $f$ and $g$ represent the respective densities of cdf's $F$ and $G$ and, for any $\mathcal{S}$, $\overline{\mathcal{S}}(x):=\mathcal{S}(\infty)-\mathcal{S}(x)$.

$$
\begin{aligned}
& .\left[\mathcal{H}_{1}\right]: \sqrt{k} A^{*}(n / k) \rightarrow d_{1}<\infty . \\
& .\left[\mathcal{H}_{2}\right]: \frac{1}{\sqrt{k}} \sum_{i=1}^{k}\left[\mathbf{p}\left(H^{\leftarrow}\left(1-\frac{i}{n}\right)\right)-p\right] \rightarrow d_{2}<\infty . \\
& .\left[\mathcal{H}_{3}\right]: \sqrt{k} \omega_{n, k}(C):=\sqrt{k} \sup _{(s, t) \in \mathcal{D}_{n}(C)}\left|\mathbf{p}\left(H^{\leftarrow}(t)\right)-\mathbf{p}\left(H^{\leftarrow}(s)\right)\right| \rightarrow 0, \text { where } \\
& \mathcal{D}_{n}(C):=\{1-k / n \leq t<1 ;|t-s| \leq C \sqrt{k} / n, s<1\}, C>0 .
\end{aligned}
$$

The authors claim that, in the case $\gamma_{1}, \gamma_{2}>0$, the function $\mathbf{p}(z)$ tends to $p$, as $z \rightarrow$ $\infty$, which, from a theoretical point of view, does not seem obvious by only assuming the regular variation of $\bar{F}$ and $\bar{G}$, hence further assumptions (like for instance the regular variation of $f$ and $g$ ) are needed. From Theorem 1.7.2 in Bingham et al. (1987) page 39, known as the Monotone Density Theorem, a necessary condition for $f$ and $g$ to be regularly varying is that $f$ and $g$ are ultimately monotone. In this paper, we give an alternative definition to $\mathbf{p}(z)$ which, among other things, avoids us additional restrictions on the underlying distributions. More precisely, we set $\mathbf{p}^{*}(z):=\bar{H}^{(1)}(z) / \bar{H}(z)$ which, from assertion (i) of Lemma 4.1, tends to $p$. With this choice and the use of the first-order regular variation conditions, we show the consistency of $\widehat{p}$, which was not addressed by Einmahl et al. (2008) who only proved its asymptotic normality under conditions $\left[\mathcal{H}_{2}\right]$ and $\left[\mathcal{H}_{3}\right]$. This result will then lead to the consistency of $\widehat{\gamma}_{1}$. On the other hand, we adopt an approach based on the empirical processes to actually provide two main results. First, we solve the problems of restriction by considering a more general family of distributions that only are regularly varying at infinity. Second, in the restricted class of distributions we relax the conditions $\left[\mathcal{H}_{1}\right]-\left[\mathcal{H}_{3}\right]$ on the sample fraction $k$ and reduce their number. To be more precise, we show that $\left[\mathcal{H}_{1}\right]-\left[\mathcal{H}_{3}\right]$ imply that $\sqrt{k}\left(\frac{n}{k} \bar{H}^{(1)}\left(U_{H}(n / k)\right)-p\right)$ tends to $d_{2}<\infty$ and that the converse is not true (see the end of Appendix).

The rest of the paper is organized a follows. In Section 2, we state our main results which consist Gaussian approximations to the adapted Hill estimator $\widehat{\gamma}_{1}$ in addition to its consistency. This contribution, which, to the best of our knowledge, is the first of its kind, will be of great usefulness in a lot of applications of extreme value theory under random censoring. The proofs are postponed to Section 3 and some results, that are instrumental to our needs, are gathered in the Appendix. 


\section{Main results}

We notice that the asymptotic normality of extreme value theory based estimators is achieved in the second-order framework. Thus, it seems quite natural to suppose that cdf's $F$ and $G$ satisfy the well-known second-order condition of regular variation. That is, we assume that there exist a constant $\tau_{j}<0$ and a function $A_{j}^{*}$, $j=1,2$, tending to zero and not changing sign near infinity, such that for any $x>0$

$$
\begin{aligned}
& \lim _{t \rightarrow \infty} \frac{U_{F}(t x) / U_{F}(t)-x^{\gamma_{1}}}{A_{1}^{*}(t)}=x^{\gamma_{1}} \frac{x^{\tau_{1}}-1}{\tau_{1}}, \\
& \lim _{t \rightarrow \infty} \frac{U_{G}(t x) / U_{G}(t)-x^{\gamma_{2}}}{A_{2}^{*}(t)}=x^{\gamma_{2}} \frac{x^{\tau_{2}}-1}{\tau_{2}} .
\end{aligned}
$$

In addition to approximating $\sqrt{k}\left(\widehat{\gamma}_{1}-\gamma_{1}\right)$, we also provide asymptotic normal representations of two other useful statistics, namely $\sqrt{k}(\widehat{p}-p)$ and $\sqrt{k}\left(Z_{n-k: n} / h-1\right)$, where

$$
h=h_{n}:=U_{H}(n / k) .
$$

For convenience, we set, for $t>1, A_{j}(t):=A_{j}^{*}(1 / \bar{F}(t)), j=1,2$.

Theorem 2.1. Assume that $\bar{F} \in \mathcal{R} \mathcal{V}_{\left(-1 / \gamma_{1}\right)}$ and $\bar{G} \in \mathcal{R} \mathcal{V}_{\left(-1 / \gamma_{2}\right)}$ and let $k=k_{n}$ be an integer sequence satisfying (1.2). Then $\widehat{\gamma}_{1} \rightarrow \gamma_{1}$ in probability. Assume further that the second-order conditions (2.5) hold and $\sqrt{k} A_{j}(h)=O(1)$, for $j=1,2$, as $n \rightarrow \infty$. Then there exists a sequence of Brownian bridges $\left\{B_{n}(s) ; 0 \leq s \leq 1\right\}$ such that

$$
\sqrt{k}\left(\frac{Z_{n-k: n}}{h}-1\right)=\gamma \sqrt{\frac{n}{k}} \mathbb{B}_{n}^{*}\left(\frac{k}{n}\right)+o_{\mathbb{P}}(1),
$$

$$
\begin{aligned}
& \sqrt{k}(\widehat{p}-p) \\
& =\sqrt{\frac{n}{k}}\left(q \mathbb{B}_{n}\left(\frac{k}{n}\right)-p \widetilde{\mathbb{B}}_{n}\left(\frac{k}{n}\right)\right)-p q\left(\frac{\gamma_{1}^{-1} \sqrt{k} A_{1}(h)}{1-p \tau_{1}}-\frac{\gamma_{2}^{-1} \sqrt{k} A_{2}(h)}{1-q \tau_{2}}\right)+o_{\mathbb{P}}(1),
\end{aligned}
$$

and

$$
\sqrt{k}\left(\widehat{\gamma}_{1}-\gamma_{1}\right)=\gamma_{1} \sqrt{\frac{n}{k}} \int_{0}^{1} s^{-1} \mathbb{B}_{n}^{*}\left(\frac{k}{n} s\right) d s-\frac{\gamma_{1}}{p} \sqrt{\frac{n}{k}} \mathbb{B}_{n}\left(\frac{k}{n}\right)+\frac{\sqrt{k} A_{1}(h)}{1-p \tau_{1}}+o_{\mathbb{P}}(1),
$$

where, $\mathbb{B}_{n}(s):=B_{n}(\theta)-B_{n}(\theta-p s)$, for $0 \leq s<\theta / p, \widetilde{\mathbb{B}}_{n}(s):=-B_{n}(1-q s)$, for $0 \leq s \leq 1$ and $\mathbb{B}_{n}^{*}(s):=\mathbb{B}_{n}(s)+\widetilde{\mathbb{B}}_{n}(s)$, for $0 \leq s<\theta / p$, are sequences of centred Gaussian processes, with $\theta:=H^{(1)}(\infty)$ and $q:=1-p$. 
Corollary 2.1. Assume that the conditions of Theorem 2.1 hold, assume further that $\sqrt{k} A_{1}(h) \rightarrow \lambda_{1}$, as $n \rightarrow \infty$. Then

$$
\sqrt{k}\left(\widehat{\gamma}_{1}-\gamma_{1}\right) \stackrel{d}{\rightarrow} \mathcal{N}\left(\frac{\lambda_{1}}{1-p \tau_{1}}, \frac{\gamma_{1}^{2}}{p}\right) \text {, as } n \rightarrow \infty
$$

where $\mathcal{N}\left(m, d^{2}\right)$ designates the normal distribution with mean $m$ and variance $d^{2}$.

Remark 2.1. Given that $p=\gamma / \gamma_{1}$, the asymptotic variance above is exactly the same as that obtained by Einmahl et al. (2008).

Theorem 2.2. Let $F$ and $G$ be two absolutely continuous cdf's with ultimately monotone densities. Assume that $\bar{F} \in \mathcal{R} \mathcal{V}_{\left(-1 / \gamma_{1}\right)}$ and $\bar{G} \in \mathcal{R} \mathcal{V}_{\left(-1 / \gamma_{2}\right)}$ and that $U_{H}$ satisfies the second-order condition (1.3). Let $k=k_{n}$ be an integer sequence such that (1.2) holds and both $\sqrt{k} A^{*}(n / k)$ and $\sqrt{k}\left(\frac{n}{k} \bar{H}^{(1)}(h)-p\right)$ are asymptotically bounded. Then

$$
\begin{gathered}
\sqrt{k}\left(\frac{Z_{n-k: n}}{h}-1\right)=\gamma \sqrt{\frac{n}{k}} \mathbb{B}_{n}^{*}\left(\frac{k}{n}\right)+o_{\mathbb{P}}(1), \\
\sqrt{k}(\widehat{p}-p)=\sqrt{\frac{n}{k}}\left(q \mathbb{B}_{n}\left(\frac{k}{n}\right)-p \widetilde{\mathbb{B}}_{n}\left(\frac{k}{n}\right)\right)+\sqrt{k}\left(\frac{n}{k} \bar{H}^{(1)}(h)-p\right)+o_{\mathbb{P}}(1),
\end{gathered}
$$

and

$$
\sqrt{k}\left(\widehat{\gamma}_{1}-\gamma_{1}\right)=\gamma_{1} \sqrt{\frac{n}{k}} \int_{0}^{1} s^{-1} \mathbb{B}_{n}^{*}\left(\frac{k}{n} s\right) d s-\frac{\gamma_{1}}{p} \sqrt{\frac{n}{k}} \mathbb{B}_{n}\left(\frac{k}{n}\right)+\sqrt{k} R_{n}+o_{\mathbb{P}}(1),
$$

where $R_{n}:=p^{-1}\left\{\frac{\gamma}{1-\tau} A^{*}(n / k)-\gamma_{1}\left(\frac{n}{k} \bar{H}^{(1)}(h)-p\right)\right\}$.

Corollary 2.2. Assume that the conditions of Theorem 2.2 hold, assume further that $\sqrt{k} A^{*}(n / k) \rightarrow d_{1}<\infty$ and $\sqrt{k}\left(\frac{n}{k} \bar{H}^{(1)}(h)-p\right) \rightarrow d_{2}<\infty$, as $n \rightarrow \infty$. Then

$$
\sqrt{k}\left(\widehat{\gamma}_{1}-\gamma_{1}\right) \stackrel{d}{\rightarrow} \mathcal{N}\left(\frac{\gamma d_{1}}{p(1-\tau)}-\frac{\gamma_{1} d_{2}}{p}, \frac{\gamma_{1}^{2}}{p}\right) \text {, as } n \rightarrow \infty
$$

Remark 2.2. Note that the asymptotic bias above agrees with that obtained by Einmahl et al. (2008). For example, when $\gamma>-\rho$, we combine relations (2.3), (2.10) and (3.4) of Fraga Alves et al. (2007) to deduce that $A^{*}(x)=\frac{\gamma(1-\tau)}{\tau+\gamma(1-\tau)} b(x)$, where $b(x)$ is a function, defined in page 214 of Einmahl et al. (2008), in terms of the convergence rate $a_{2}(x)$ of the second-order condition, given in (9). Now, observe that $\left[\mathcal{H}_{1}\right]$ is expressed as $\sqrt{k} b(n / k) \rightarrow \alpha_{1}$ and that the constant $d_{2}$ in $\left[\mathcal{H}_{2}\right]$ is denoted by $\alpha_{2}$, in the second assumption, in Einmahl et al. (2008). This leads, after substitution, to the same bias. 


\section{Proofs}

We begin by a brief introduction on some uniform empirical processes under random censoring. The empirical counterparts of $H^{(j)}(j=0,1)$ and the pertaining empirical processes are respectively defined, for $v \geq 0$, by

$$
H_{n}^{(j)}(v):=\frac{1}{n} \sum_{i=1}^{n} \mathbf{1}\left(Z_{i} \leq v, \delta_{i}=j\right) \text { and } \sqrt{n}\left(\bar{H}_{n}^{(j)}(v)-\bar{H}^{(j)}(v)\right), j=0,1 .
$$

The latter may be represented, almost surely, by a uniform empirical process. Indeed, let us define $U_{i}:=\delta_{i} H^{(1)}\left(Z_{i}\right)+\left(1-\delta_{i}\right)\left(\theta+H^{(0)}\left(Z_{i}\right)\right), i=1, \ldots, n$. From Einmahl and Koning (1992), the rv's $U_{1}, \ldots, U_{n}$ are iid $(0,1)$-uniform. The empirical cdf and the uniform empirical process based upon $U_{1}, \ldots, U_{n}$ are respectively denoted by

$$
\mathbb{U}_{n}(s):=\frac{1}{n} \sum_{i=1}^{n} \mathbf{1}\left(U_{i} \leq s\right) \text { and } \alpha_{n}(s):=\sqrt{n}\left(\mathbb{U}_{n}(s)-s\right), 0 \leq s \leq 1 .
$$

We have almost surely $H_{n}^{(0)}(v)=\mathbb{U}_{n}\left(H^{(0)}(v)+\theta\right)-\mathbb{U}_{n}(\theta)$, for $0<H^{(0)}(v)<1-\theta$ and $H_{n}^{(1)}(v)=\mathbb{U}_{n}\left(H^{(1)}(v)\right)$, for $0<H^{(1)}(v)<\theta$ (see Deheuvels and Einmahl, 1996). It is easy to verify that almost surely

$$
\sqrt{n}\left(\bar{H}_{n}^{(1)}(v)-\bar{H}^{(1)}(v)\right)=\alpha_{n}(\theta)-\alpha_{n}\left(\theta-\bar{H}^{(1)}(v)\right), \text { for } 0<\bar{H}^{(1)}(v)<\theta,
$$

and

$$
\sqrt{n}\left(\bar{H}_{n}^{(0)}(v)-\bar{H}^{(0)}(v)\right)=-\alpha_{n}\left(1-\bar{H}^{(0)}(v)\right), \text { for } 0<\bar{H}^{(0)}(v)<1-\theta .
$$

Our methodology strongly relies on the well-known Gaussian approximation, given by Csörgö et al. (1986) by in Corollary 2.1, which says that on the probability space $(\Omega, \mathcal{A}, \mathbb{P})$, there exists a sequence of Brownian bridges $\left\{B_{n}(s) ; 0 \leq s \leq 1\right\}$ such that for every $0 \leq \xi<1 / 4$,

$$
\sup _{1 / n \leq s \leq 1-1 / n} \frac{n^{\xi}\left|\alpha_{n}(s)-B_{n}(s)\right|}{[s(1-s)]^{1 / 2-\xi}}=O_{\mathbb{P}}(1) .
$$

For the increments $\alpha_{n}(\theta)-\alpha_{n}(\theta-s)$, we will need an approximation of the same type as (3.12). Following similar arguments, mutatis mutandis, as those used to in the proof of assertions (2.2) of Theorem 2.1 and (2.8) of Theorem 2.2 in Csörgö et al. (1986), we may show that, for every $0<\theta<1$ and $0 \leq \xi<1 / 4$, we have

$$
\sup _{1 / n \leq s \leq \theta} \frac{n^{\xi}\left|\left\{\alpha_{n}(\theta)-\alpha_{n}(\theta-s)\right\}-\left\{B_{n}(\theta)-B_{n}(\theta-s)\right\}\right|}{s^{1 / 2-\xi}}=O_{\mathbb{P}}(1) .
$$


The following processes will be crucial to our needs:

$$
\beta_{n}(v):=\sqrt{\frac{n}{k}}\left\{\alpha_{n}(\theta)-\alpha_{n}\left(\theta-\bar{H}^{(1)}\left(Z_{n-k: n} v\right)\right)\right\}, \text { for } 0<\bar{H}^{(1)}(v)<\theta,
$$

and

$$
\widetilde{\beta}_{n}(v):=-\sqrt{\frac{n}{k}} \alpha_{n}\left(1-\bar{H}^{(0)}\left(Z_{n-k: n} v\right)\right), \text { for } 0<\bar{H}^{(0)}(v)<1-\theta .
$$

3.1. Proof of Theorem 2.1. We start by showing the consistency of estimator $\widehat{p}$. After observing that $\widehat{p}=\frac{n}{k} \bar{H}_{n}^{(1)}\left(Z_{n-k: n}\right)$, we consider the following decomposition:

$$
\begin{aligned}
\widehat{p}-p & =\frac{n}{k}\left(\bar{H}_{n}^{(1)}\left(Z_{n-k: n}\right)-\bar{H}^{(1)}\left(Z_{n-k: n}\right)\right) \\
& +\frac{n}{k}\left(\bar{H}^{(1)}\left(Z_{n-k: n}\right)-\bar{H}^{(1)}(h)\right)+\left(\frac{n}{k} \bar{H}^{(1)}(h)-p\right) .
\end{aligned}
$$

Note that, from (3.14), we have almost surely

$$
\sqrt{k} \frac{n}{k}\left(\bar{H}_{n}^{(1)}\left(Z_{n-k: n}\right)-\bar{H}^{(1)}\left(Z_{n-k: n}\right)\right)=\beta_{n}(1) .
$$

By using the Gaussian approximation (3.12), we get

$$
\sqrt{k} \frac{n}{k}\left(\bar{H}_{n}^{(1)}\left(Z_{n-k: n}\right)-\bar{H}^{(1)}\left(Z_{n-k: n}\right)\right)=\sqrt{n / k} \mathbf{B}_{n}\left(Z_{n-k: n}\right)+o_{\mathbb{P}}(1),
$$

where

$$
\mathbf{B}_{n}(v):=B_{n}(\theta)-B_{n}\left(\theta-\bar{H}^{(1)}(v)\right) \text {, for } 0<\bar{H}^{(1)}(v)<\theta .
$$

Making use of Lemma 4.2 , we infer that $\beta_{n}(1)=\sqrt{n / k} \mathbb{B}_{n}(k / n)+o_{\mathbb{P}}(1)$. It is easy to verify that $\sqrt{n / k} \mathbb{B}_{n}(k / n)$ is asymptotically centred Gaussian rv with variance $p$, it follows that the first term in the right-hand side of $(3.16)$ is $O_{\mathbb{P}}\left(k^{-1 / 2}\right)$ which tends to zero in probability. The second term in the right-hand side of (3.16) may be written as

$$
\frac{n}{k}\left(\bar{H}^{(1)}\left(Z_{n-k: n}\right)-\bar{H}^{(1)}(h)\right)=\frac{n}{k} \bar{H}^{(1)}(h)\left(\frac{\bar{H}^{(1)}\left(Z_{n-k: n}\right)}{\bar{H}^{(1)}(h)}-1\right) .
$$

Since $\bar{H}$ is regularly varying at infinity with index $(-1 / \gamma)$, then by letting $v=1$ in part (i) of Lemma 4.1, it is easy to verify that $\bar{H}^{(1)}$ is also regularly varying with the same index. First, we note that, combining Corollary 2.2.2 with Potter's inequalities given in Proposition B.1.9 (5) in de Haan and Ferreira (2006), yields that $Z_{n-k: n} / h \rightarrow 1$ in probability. Now, we use Potter's inequalities (see de Haan and Ferreira, 2006, Proposition B.1.9 (5)) with the fact that $Z_{n-k: n} / h \rightarrow 1$ in probability, to show that $\bar{H}^{(1)}\left(Z_{n-k: n}\right) / \bar{H}^{(1)}(h)-1 \rightarrow 0$, in probability. If, in addition to $v=1$, we take $z=h$ in part (i) of Lemma 4 .1, then we get $n \bar{H}^{(1)}(h) / k \rightarrow p$ 
and therefore the second term in the right-hand side of (3.16) tends to zero in probability as well. Finally, noting that the third term in the right-hand side of (3.16) clearly goes to zero, yields that $\widehat{p}-p$ in probability as sought.

For result (2.7), we write

$$
\frac{\bar{H}(t x)}{\bar{H}(t)}-x^{-1 / \gamma}=\frac{\bar{G}(t x)}{\bar{G}(t)} \frac{\frac{\bar{F}(t x)}{\bar{F}(t)}-x^{-1 / \gamma_{1}}}{A_{1}(t)} A_{1}(t)+x^{-1 / \gamma_{1}} \frac{\frac{\bar{G}(t x)}{\bar{G}(t)}-x^{-1 / \gamma_{2}}}{A_{2}(t)} A_{2}(t) .
$$

From Theorem 2.3.9 in de Haan and Ferreira (2006), page 48, the first and the second conditions in (2.5) are respectively equivalent to

$$
\frac{\frac{\bar{F}(t x)}{\bar{F}(t)}-x^{-1 / \gamma_{1}}}{A_{1}(t)} \rightarrow x^{-1 / \gamma_{1}} \frac{x^{\tau_{1} / \gamma_{1}}-1}{\gamma_{1} \tau_{1}} \text { and } \frac{\overline{\bar{G}(t x)}-x^{-1 / \gamma_{2}}}{A_{2}(t)} \rightarrow x^{-1 / \gamma_{2}} \frac{x^{\tau_{2} / \gamma_{2}}-1}{\gamma_{2} \tau_{2}},
$$

as $t \rightarrow \infty$. This implies that

$$
\frac{\bar{H}(t x)}{\bar{H}(t)}-x^{-1 / \gamma} \sim x^{-1 / \gamma} \frac{x^{\tau_{1} / \gamma_{1}}-1}{\gamma_{1} \tau_{1}} A_{1}(t)+x^{-1 / \gamma} \frac{x^{\tau_{2} / \gamma_{2}}-1}{\gamma_{2} \tau_{2}} A_{2}(t) .
$$

In the sequel, for two sequences of rv's, we write $V_{n}^{(1)}=o_{\mathbb{P}}\left(V_{n}^{(2)}\right)$, as $n \rightarrow \infty$, to say that $V_{n}^{(1)} / V_{n}^{(2)} \rightarrow 0$ in probability. Let now $x=x_{n}=Z_{n-k: n} / h$ and $t=t_{n}=h$. Since $x_{n}=1+o_{\mathbb{P}}(1)$, then $\frac{x_{n}^{-\tau_{i} / \gamma_{i}}-1}{\tau_{i} \gamma_{i}}, i=1,2$, tend to zero in probability and therefore by using (3.20), we get

$$
\left(\frac{Z_{n-k: n}}{h}\right)^{-1 / \gamma}=\frac{\bar{H}\left(Z_{n-k: n}\right)}{\bar{H}(h)}+o_{\mathbb{P}}\left(A_{1}(h)+A_{2}(h)\right) .
$$

But $\bar{H}(h)=k / n$, then

$$
\left(\frac{Z_{n-k: n}}{h}\right)^{-1 / \gamma}-1=\frac{\bar{H}\left(Z_{n-k: n}\right)}{k / n}-1+o_{\mathbb{P}}\left(A_{1}(h)+A_{2}(h)\right) .
$$

Applying the mean value theorem to the left-hand side yields

$$
-\frac{1}{\gamma}\left(\frac{Z_{n-k: n}}{h}-1\right) c_{n}^{-1 / \gamma-1}=\frac{\bar{H}\left(Z_{n-k: n}\right)}{k / n}-1+o_{\mathbb{P}}\left(A_{1}(h)+A_{2}(h)\right),
$$

where $c_{n}$ is a sequence of rv's lying between 1 and $Z_{n-k: n} / h$, meaning that we have $c_{n}=1+o_{\mathbb{P}}(1)$. It follows that

$$
\frac{Z_{n-k: n}}{h}-1=-\left(1+o_{\mathbb{P}}(1)\right) \gamma\left(\frac{\bar{H}\left(Z_{n-k: n}\right)}{k / n}-1\right)+o_{\mathbb{P}}\left(A_{1}(h)+A_{2}(h)\right) .
$$

By assumption, we have $\sqrt{k} A_{j}(h)=O(1), j=1,2$, then

$$
\sqrt{k}\left(\frac{Z_{n-k: n}}{h}-1\right)=\left(1+o_{\mathbb{P}}(1)\right) \gamma \sqrt{k} \frac{n}{k}\left(k / n-\bar{H}\left(Z_{n-k: n}\right)\right)+o_{\mathbb{P}}(1) .
$$


10

Recall that $\bar{H}_{n}\left(Z_{n-k: n}\right)=k / n$, then

$$
\sqrt{k}\left(\frac{Z_{n-k: n}}{h}-1\right)=\left(1+o_{\mathbb{P}}(1)\right) \gamma \sqrt{k} \frac{n}{k}\left(\bar{H}_{n}\left(Z_{n-k: n}\right)-\bar{H}\left(Z_{n-k: n}\right)\right)+o_{\mathbb{P}}(1) .
$$

The right-hand side of the previous equation may be decomposed into

$$
\begin{aligned}
\gamma \sqrt{k} \frac{n}{k} & \left(\bar{H}_{n}^{(1)}\left(Z_{n-k: n}\right)-\bar{H}^{(1)}\left(Z_{n-k: n}\right)\right) \\
& \quad+\gamma \sqrt{k} \frac{n}{k}\left(\bar{H}_{n}^{(0)}\left(Z_{n-k: n}\right)-\bar{H}^{(0)}\left(Z_{n-k: n}\right)\right)\left(1+o_{\mathbb{P}}(1)\right)+o_{\mathbb{P}}(1) .
\end{aligned}
$$

Using (3.14) and (3.15) with $v=1$, leads to

$$
\sqrt{k}\left(\frac{Z_{n-k: n}}{h}-1\right)=\gamma\left(\beta_{n}(1)+\widetilde{\beta}_{n}(1)\right)\left(1+o_{\mathbb{P}}(1)\right)+o_{\mathbb{P}}(1)
$$

which, by the Gaussian representations (3.12) and (3.13), becomes

$$
\sqrt{k}\left(\frac{Z_{n-k: n}}{h}-1\right)=\gamma \sqrt{\frac{n}{k}} \mathbf{B}_{n}^{*}\left(Z_{n-k: n}\right)\left(1+o_{\mathbb{P}}(1)\right)+o_{\mathbb{P}}(1),
$$

where

$$
\mathbf{B}_{n}^{*}(v):=\mathbf{B}_{n}(v)-B_{n}\left(1-\bar{H}^{(0)}(v)\right) \text {, for } 0<\bar{H}^{(0)}(v)<1-\theta,
$$

with $\mathbf{B}_{n}(v)$ defined in (3.18). Finally, we use assertion ( $\left.i i\right)$ of Lemma 4.2 to complete the proof of result (2.7). For result (2.8), we multiply decomposition (3.16) by $\sqrt{k}$ and get

$$
\begin{aligned}
\sqrt{k}(\widehat{p}-p) & =\sqrt{k} \frac{n}{k}\left(\bar{H}_{n}^{(1)}\left(Z_{n-k: n}\right)-\bar{H}^{(1)}\left(Z_{n-k: n}\right)\right) \\
& +\sqrt{k} \frac{n}{k}\left(\bar{H}^{(1)}\left(Z_{n-k: n}\right)-\bar{H}^{(1)}(h)\right)+\sqrt{k}\left(\frac{n}{k} \bar{H}^{(1)}(h)-p\right) .
\end{aligned}
$$

Next, we represent the first two terms, of the right-side hand of the previous equation, by $\beta_{n}$ and $\widetilde{\beta}_{n}$ and then we use approximations (3.12) and (3.13). Recall that, from (3.17), the first one has already been shown to be equal to $\beta_{n}(1)$ almost surely. For the second one, we use (3.19) and assertion (ii) of Lemma 4.1 to have

$$
\frac{n}{k}\left(\bar{H}^{(1)}\left(Z_{n-k: n}\right)-\bar{H}^{(1)}(h)\right)=p\left\{\left(\frac{Z_{n-k: n}}{h}\right)^{-1 / \gamma}-1\right\}+o_{\mathbb{P}}\left(A_{1}(h)+A_{2}(h)\right) .
$$

By applying the mean value theorem and using the fact that $Z_{n-k: n} / h=1+o_{\mathbb{P}}(1)$, we readily verify that $\left(Z_{n-k: n} / h\right)^{-1 / \gamma}-1=\left(1+o_{\mathbb{P}}(1)\right) \gamma^{-1}\left(1-Z_{n-k: n} / h\right)$. Hence

$$
\begin{aligned}
& \frac{n}{k}\left(\bar{H}^{(1)}\left(Z_{n-k: n}\right)-\bar{H}^{(1)}(h)\right) \\
& =-\frac{p}{\gamma}\left(\frac{Z_{n-k: n}}{h}-1\right)\left(1+o_{\mathbb{P}}(1)\right)+o_{\mathbb{P}}\left(A_{1}(h)+A_{2}(h)\right) .
\end{aligned}
$$


Using the assumptions $\sqrt{k} A_{j}(h)=O(1), j=1,2$, and (3.22), we obtain

$$
\sqrt{k} \frac{n}{k}\left(\bar{H}^{(1)}\left(Z_{n-k: n}\right)-\bar{H}^{(1)}(h)\right)=-p\left(\beta_{n}(1)+\widetilde{\beta}_{n}(1)\right)+o_{\mathbb{P}}(1) .
$$

Now, we use result (ii) of Lemma 4.1 with the fact that $\sqrt{k} A_{j}(h)=O(1), j=1,2$, to rewrite the third term in the right-hand side of (3.25) into

$$
\sqrt{k}\left(\frac{n}{k} \bar{H}^{(1)}(h)-p\right)=\sqrt{k} b_{1}(h)+o(1) .
$$

Substituting results (3.17), (3.27) and (3.28) in decomposition (3.16), yields

$$
\sqrt{k}(\widehat{p}-p)=\beta_{n}(1)-p\left(\beta_{n}(1)+\widetilde{\beta}_{n}(1)\right)+\sqrt{k} b_{1}(h)+o_{\mathbb{P}}(1),
$$

and the Gaussian approximations (3.12) and (3.13) imply that

$$
\sqrt{k}(\widehat{p}-p)=\sqrt{\frac{n}{k}}\left(\mathbf{B}_{n}\left(Z_{n-k: n}\right)-p \mathbf{B}_{n}^{*}\left(Z_{n-k: n}\right)\right)+\sqrt{k} b_{1}(h)+o_{\mathbb{P}}(1) .
$$

The final form of result (2.8) is then obtained by applying assertions $(i)$ and $(i i)$ of Lemma 4.2. Finally, we focus on (2.9), which represents the main result of Theorem 2.1. It is readily checked that we have

$$
\sqrt{k}\left(\widehat{\gamma}_{1}-\gamma_{1}\right)=\frac{1}{\widehat{p}} \sqrt{k}\left(\widehat{\gamma}^{H}-\gamma\right)-\frac{\gamma_{1}}{\widehat{p}} \sqrt{k}(\widehat{p}-p) .
$$

Recall that one way to define Hill's estimator $\widehat{\gamma}^{H}$ is to use the limit

$$
\gamma=\lim _{t \rightarrow \infty} \int_{t}^{\infty} v^{-1} \frac{\bar{H}(v)}{\bar{H}(t)} d v .
$$

Then, by replacing $\bar{H}$ by $\bar{H}_{n}$ and letting $t=Z_{n-k: n}$, we write

$$
\widehat{\gamma}^{H}=\frac{n}{k} \int_{Z_{n-k: n}}^{\infty} v^{-1} \bar{H}_{n}(v) d v .
$$

For details, see for instance, de Haan and Ferreira (2006) page 69. Writing $\bar{H}_{n}(v)$ as the sum of $\bar{H}_{n}^{(0)}(v)$ and $\bar{H}_{n}^{(1)}(v)$, we decompose $\hat{\gamma}^{H}-\gamma$ into the sum of the following three terms

$$
\begin{aligned}
T_{n 1} & :=\frac{n}{k} \int_{Z_{n-k: n}}^{\infty} v^{-1}\left(\bar{H}_{n}^{(0)}(v)-\bar{H}^{(0)}(v)+\bar{H}_{n}^{(1)}(v)-\bar{H}^{(1)}(v)\right) d v, \\
T_{n 2} & :=\frac{n}{k} \int_{Z_{n-k: n}}^{h} v^{-1} \bar{H}(v) d v \text { and } T_{n 3}:=\frac{n}{k} \int_{h}^{\infty} v^{-1} \bar{H}(v) d v-\gamma .
\end{aligned}
$$

Making a change of variables in the first term $T_{n 1}$ and using (3.10), (3.11), (3.14) and (3.15), we get almost surely

$$
\sqrt{k} T_{n 1}=\int_{1}^{\infty} v^{-1}\left(\beta_{n}(v)+\widetilde{\beta}_{n}(v)\right) d v
$$


For the second term $T_{n 2}$, we apply the mean value theorem to have

$$
T_{n 2}=\frac{n}{k} \frac{\bar{H}\left(z_{n}^{*}\right)}{z_{n}^{*}}\left(h-Z_{n-k: n}\right),
$$

where $z_{n}^{*}$ is a sequence of rv's lying between $Z_{n-k: n}$ and $h$. It is obvious that we have $z_{n}^{*}=\left(1+o_{\mathbb{P}}(1)\right) h$, this implies that $\bar{H}\left(z_{n}^{*}\right)=\left(1+o_{\mathbb{P}}(1)\right) k / n$. It follows that the right-hand side of the previous equation is equal to $\left(1+o_{\mathbb{P}}(1)\right)\left(1-Z_{n-k: n} / h\right)$. Hence, from (3.22), we have

$$
\sqrt{k} T_{n 2}=-\gamma\left(\beta_{n}(1)+\widetilde{\beta}_{n}(1)\right)+o_{\mathbb{P}}(1) .
$$

Finally, for $T_{n 3}$, we use the second-order conditions (2.5) to get

$$
\sqrt{k} T_{n 3} \sim p^{2} \frac{\sqrt{k} A_{1}(h)}{1-p \tau_{1}}+q^{2} \frac{\sqrt{k} A_{2}(h)}{1-q \tau_{2}}=: \sqrt{k} b_{2}(h) .
$$

Since by assumption $\sqrt{k} A_{j}(h)=O(1), j=1,2$, as $n \rightarrow \infty$, then

$$
\begin{aligned}
& \sqrt{k}\left(\hat{\gamma}^{H}-\gamma\right) \\
& =\int_{1}^{\infty} v^{-1}\left(\beta_{n}(v)+\widetilde{\beta}_{n}(v)\right) d v-\gamma\left(\beta_{n}(1)+\widetilde{\beta}_{n}(1)\right)+\sqrt{k} b_{2}(h)+o_{\mathbb{P}}(1) .
\end{aligned}
$$

Combining (3.29) and (3.31) with (3.30) yields

$$
\begin{aligned}
& \sqrt{k}\left(\widehat{\gamma}_{1}-\gamma_{1}\right) \\
& =\frac{1}{p} \int_{1}^{\infty} v^{-1}\left(\beta_{n}(v)+\widetilde{\beta}_{n}(v)\right) d v-\frac{\gamma_{1}}{p} \beta_{n}(1)+\sqrt{k} b(h)+o_{\mathbb{P}}(1),
\end{aligned}
$$

where

$$
b(h):=\frac{1}{p} b_{2}(h)-\frac{\gamma_{1}}{p} b_{1}(h)=\frac{A_{1}(h)}{1-p \tau_{1}} .
$$

Once again, by using the Gaussian approximations (3.12) and (3.13), we obtain

$$
\begin{aligned}
& \sqrt{k}\left(\widehat{\gamma}_{1}-\gamma_{1}\right) \\
& =\frac{1}{p} \sqrt{\frac{n}{k}} \int_{1}^{\infty} v^{-1} \mathbf{B}_{n}^{*}\left(v Z_{n-k: n}\right) d v-\frac{\gamma_{1}}{p} \sqrt{\frac{n}{k}} \mathbf{B}_{n}\left(Z_{n-k: n}\right)+\sqrt{k} b(k)+o_{\mathbb{P}}(1),
\end{aligned}
$$

which, by using assertions $(i)$ and $(i i i)$ of Lemma 4.2, may be rewritten into

$$
\begin{aligned}
& \sqrt{k}\left(\widehat{\gamma}_{1}-\gamma_{1}\right) \\
& =\frac{\gamma}{p} \sqrt{\frac{n}{k}} \int_{0}^{1} s^{-1} \mathbb{B}_{n}^{*}\left(\frac{k}{n} s\right) d s-\frac{\gamma_{1}}{p} \sqrt{\frac{n}{k}} \mathbb{B}_{n}\left(\frac{k}{n}\right)+\sqrt{k} b(h)+o_{\mathbb{P}}(1) .
\end{aligned}
$$

Actually, applying the Gaussian approximations (3.12) and (3.13) here needs some usual manipulation on the upper bound of integration as recently done in Brahimi et al. (2013), we omit details. Replacing $\gamma$ by $p \gamma_{1}$ in the front of the integral and using (3.32) achieve the proof of the third result of Theorem 2.1. 
3.2. Proof of Corollary 2.1. From result (2.9) of Theorem 2.1, we deduce that $\sqrt{k}\left(\widehat{\gamma}_{1}-\gamma_{1}\right)$ is asymptotically Gaussian with mean $\frac{\lambda_{1}}{1-p \tau_{1}}$, and variance

$$
\gamma_{1}^{2} \lim _{n \rightarrow \infty} \mathbf{E}\left[\sqrt{\frac{n}{k}} \int_{0}^{1} s^{-1} \mathbb{B}_{n}^{*}\left(\frac{k}{n} s\right) d s-\frac{1}{p} \sqrt{\frac{n}{k}} \mathbb{B}_{n}\left(\frac{k}{n}\right)\right]^{2} .
$$

We check that the processes $\mathbb{B}_{n}(s), \widetilde{\mathbb{B}}_{n}(s)$ and $\mathbb{B}_{n}^{*}(s)$ satisfy $p^{-1} \mathbf{E}\left[\mathbb{B}_{n}(s) \mathbb{B}_{n}(t)\right]=$ $\min (s, t)-p s t, q^{-1} \mathbf{E}\left[\widetilde{\mathbb{B}}_{n}(s) \widetilde{\mathbb{B}}_{n}(t)\right]=\min (s, t)-q s t$ and $p^{-1} \mathbf{E}\left[\mathbb{B}_{n}(s) \mathbb{B}_{n}^{*}(t)\right]=$ $\mathbf{E}\left[\mathbb{B}_{n}^{*}(s) \mathbb{B}_{n}^{*}(t)\right]=\min (s, t)-s t$. Then, by elementary calculation (we omit details), we obtain $\gamma_{1}^{2} / p$ for the asymptotic variance of $\sqrt{k}\left(\widehat{\gamma}_{1}-\gamma_{1}\right)$, which, since $p=\gamma / \gamma_{1}$, is equal to $\gamma_{1}^{3} / \gamma$.

\subsection{Proof of Theorem 2.2. Recall that}

$$
\sqrt{k}\left(\widehat{\gamma}_{1}-\gamma_{1}\right)=\frac{1}{\widehat{p}} \sqrt{k}\left(\widehat{\gamma}^{H}-\gamma\right)-\frac{\gamma_{1}}{\widehat{p}} \sqrt{k}(\widehat{p}-p)
$$

For the first term, we use the Gaussian approximation (3.31) with a bias equal to $\sqrt{k} \frac{\gamma}{p(1-\tau)} A^{*}(n / k)$ under the assumption that $\sqrt{k} A^{*}(n / k)$ is bounded. Now we consider $\sqrt{k}(\widehat{p}-p)$. The third term of the right-hand side of (3.16) is assumed to be asymptotically bounded while the first one is already approximated by a Gaussian process by only being in the first-order framework of regular variation. For the second term, denoted by $\Omega_{n}$, we apply the mean value theorem to get

$$
\Omega_{n}=\frac{n}{k} h\left(Z_{n-k: n} / h-1\right)\left(\bar{H}^{(1)}\right)^{\prime}\left(\eta_{n}\right),
$$

with $\eta_{n}$ between $h$ and $Z_{n-k: n}$. Since $\bar{F} \in \mathcal{R} \mathcal{V}_{\left(-1 / \gamma_{1}\right)}$ with $f$ ultimately monotone, then $f \in \mathcal{R} \mathcal{V}_{\left(-1 / \gamma_{1}-1\right)}$ by Theorem 1.7.2 in Bingham et al. (1987) page 39, it follows that $z\left(\bar{H}^{(1)}\right)^{\prime}(z) \sim-\gamma_{1}^{-1} \bar{H}(z)$. From Potter's inequalities and the fact that $Z_{n-k: n} / h=1+o_{\mathbb{P}}(1)$, we infer that

$$
h\left(\bar{H}^{(1)}\right)^{\prime}\left(\eta_{n}\right)=-\left(1+o_{\mathbb{P}}(1)\right) \gamma_{1}^{-1} \bar{H}(h) .
$$

Observe that $k / n=\bar{H}(h)$, then

$$
\Omega_{n}=-\left(1+o_{\mathbb{P}}(1)\right) \gamma_{1}^{-1}\left(Z_{n-k: n} / h-1\right),
$$

which coincides with the first term in the right-hand side of (3.26). From this point on, we proceed as in the proof of Theorem 2.1 to complete the proof.

\section{Concluding notes}

The primary object of the present work consists in providing a Gaussian limiting distribution for the estimator of the shape parameter of a heavy-tailed distribution, 
under random censorship. Our methodology is based on the approximation of the uniform empirical process by a sequence of Brownian bridges. This approach relaxes the assumptions imposed in Theorem 1 of Einmahl et al. (2008) and reduces their number. It is noteworthy that, for $p=1$ (non censoring case), our main result perfectly agrees with the Gaussian representation of the classical Hill estimator. The generalization of this approximating procedure to the moment estimator (valid for any real-valued EVI) adapted to random censorship in Einmahl et al. (2008), will be considered in a future work.

\section{Appendix}

Lemma 4.1. (i) Assume that $\bar{F} \in \mathcal{R}_{\left(-1 / \gamma_{1}\right)}$ and $\bar{G} \in \mathcal{R} \mathcal{V}_{\left(-1 / \gamma_{2}\right)}$ and let $k:=k_{n}$ be an integer sequence satisfying (1.2). Then

$$
\lim _{z \rightarrow \infty} \sup _{v \geq 1}\left|\bar{H}^{(1)}(z v) / \bar{H}(z)-p v^{-1 / \gamma}\right|=0
$$

(ii) If further the second-order conditions of regular variation (2.5) hold, then we have, uniformly on $v \geq 1$,

$$
v^{1 / \gamma} \frac{n}{k} \bar{H}^{(1)}(h v)=p+b_{1}(v ; h)+o\left(A_{1}(h)+A_{2}(h)\right), \text { as } n \rightarrow \infty,
$$

where

$$
b_{1}(v ; h):=\frac{p}{\gamma_{1} \tau_{1}}\left(\frac{1-\tau_{1}}{1-p \tau_{1}} v^{\tau_{1} / \gamma_{1}}-1\right) A_{1}(h)+\frac{p}{\gamma_{2} \tau_{2}}\left(\frac{1}{1-q \tau_{2}} v^{\tau_{2} / \gamma_{2}}-1\right) A_{2}(h) .
$$

Proof. It is easy to verify that, for $v \geq 1$, we have $H^{(1)}(v)=\int_{0}^{v} \bar{G}(y) d F(y)$ and

$$
\frac{\bar{H}^{(1)}(z v)}{\bar{H}(z)}=-\int_{v}^{\infty} \frac{\bar{G}(h x)}{\bar{G}(h)} d \frac{\bar{F}(h x)}{\bar{F}(h)} .
$$

To prove (i), we directly use Proposition B.1.10 in de Haan and Ferreira (2006) page 369 to the regularly varying functions $\bar{F}$ and $\bar{G}$ : for any $0<\epsilon<1$, there exists $n_{0}=n_{0}(\epsilon)$, such that for all $n>n_{0}$ and $x \geq 1$,

$$
\left|\frac{\bar{F}(h x)}{\bar{F}(h)}-x^{-1 / \gamma_{1}}\right|<\epsilon x^{-1 / \gamma_{1}+\epsilon} \text { and }\left|\frac{\bar{G}(h x)}{\bar{G}(h)}-x^{-1 / \gamma_{2}}\right|<\epsilon x^{-1 / \gamma_{2}+\epsilon} .
$$

For (ii), we use the uniform inequalities (for the second-order regularly varying functions) to both tails $\bar{F}$ and $\bar{G}$ (see, e.g., de Haan and Ferreira, 2006, bottom of page 161): for any $0<\epsilon<1$, there exists $n_{0}=n_{0}(\epsilon)$, such that for all $n>n_{0}$ and $x \geq 1$

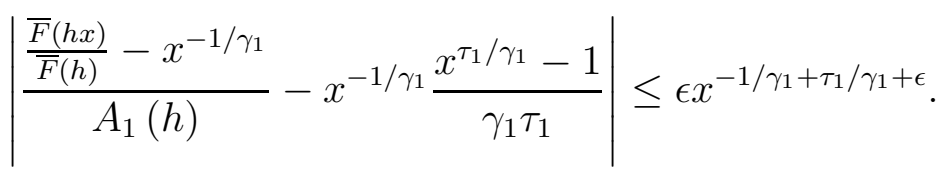


and

$$
\left|\frac{\frac{\bar{G}(h x)}{\bar{G}(h)}-x^{-1 / \gamma_{2}}}{A_{2}(h)}-x^{-1 / \gamma_{2}} \frac{x^{\tau_{2} / \gamma_{2}}-1}{\gamma_{2} \tau_{2}}\right| \leq \epsilon x^{-1 / \gamma_{2}+\tau_{2} / \gamma_{2}+\epsilon} .
$$

Therefore we omit details.

Lemma 4.2. Under the assumptions of assertion (i) in Lemma 4.1, we have

$$
\begin{aligned}
& \text { (i) } \sqrt{\frac{n}{k}} \mathbf{B}_{n}\left(v Z_{n-k: n}\right)=\sqrt{\frac{n}{k}} \mathbb{B}_{n}\left(\frac{k}{n} v^{-1 / \gamma}\right)+o_{\mathbb{P}}(1), \text { for every } v \geq 1, \\
& \text { (ii) } \sqrt{\frac{n}{k}} \mathbf{B}_{n}^{*}\left(v Z_{n-k: n}\right)=\sqrt{\frac{n}{k}} \mathbb{B}_{n}^{*}\left(\frac{k}{n} v^{-1 / \gamma}\right)+o_{\mathbb{P}}(1), \text { for every } v \geq 1, \\
& \text { (iii) } \sqrt{\frac{n}{k}} \int_{1}^{\infty} v^{-1} \mathbf{B}_{n}^{*}\left(v Z_{n-k: n}\right) d v=\gamma \sqrt{\frac{n}{k}} \int_{0}^{1} s^{-1} \mathbb{B}_{n}^{*}\left(\frac{k}{n} s\right) d s+o_{\mathbb{P}}(1) .
\end{aligned}
$$

Proof. First, note that we will omit the details for assertion (ii), as it is shown by similar arguments than those used to prove $(i)$. For this latter, we have to show that for a fixed $v \geq 1$,

$$
\sqrt{\frac{n}{k}}\left\{B_{n}\left(\theta-\bar{H}^{(1)}\left(Z_{n-k: n} v\right)\right)-B_{n}\left(\theta-p \frac{k}{n} v^{-1 / \gamma}\right)\right\}=o_{\mathbb{P}}(1) .
$$

Indeed, let $\left\{W_{n}(t) ; 0 \leq t \leq 1\right\}$ be a sequence of Wiener processes defined on $(\Omega, \mathcal{A}, \mathbb{P})$ so that

$$
\left\{B_{n}(t) ; 0 \leq t \leq 1\right\} \stackrel{d}{=}\left\{W_{n}(t)-t W_{n}(1) ; 0 \leq t \leq 1\right\}
$$

Then, without loss of generality, we may write

$$
\begin{gathered}
\sqrt{\frac{n}{k}}\left\{B_{n}\left(\theta-\bar{H}^{(1)}\left(Z_{n-k: n} v\right)\right)-B_{n}\left(\theta-p \frac{k}{n} v^{-1 / \gamma}\right)\right\} \\
=\sqrt{\frac{n}{k}}\left\{W_{n}\left(\theta-\bar{H}^{(1)}\left(Z_{n-k: n} v\right)\right)-W_{n}\left(\theta-p \frac{k}{n} v^{-1 / \gamma}\right)\right\} \\
-\sqrt{\frac{n}{k}}\left(p \frac{k}{n} v^{-1 / \gamma}-\bar{H}^{(1)}\left(Z_{n-k: n} v\right)\right) W_{n}(1) .
\end{gathered}
$$

Next, we show that both terms in the right-hand side tend to zero (in probability), as $n \rightarrow \infty$. Let $v \geq 1$ be fixed and recall that $\bar{H}^{(1)}\left(Z_{n-k: n} v\right)=\left(1+o_{\mathbb{P}}(1)\right) p v^{-1 / \gamma} k / n$, then the result follows for the second term. For the first one, we have, for given $0<\eta, \epsilon<1$ (small enough) and for all large $n$,

$$
\mathbb{P}\left(\left|\frac{\bar{H}^{(1)}\left(Z_{n-k: n} v\right)}{v^{-1 / \gamma} k / n}-p\right|>\eta^{2} \frac{\epsilon^{2}}{4 v^{1 / \gamma}}\right)<\epsilon / 2 .
$$


Observe now that

$$
\begin{aligned}
& \mathbb{P}\left(\sqrt{\frac{n}{k}}\left|W_{n}\left(\theta-\bar{H}^{(1)}\left(Z_{n-k: n} v\right)\right)-W_{n}\left(\theta-p \frac{k}{n} v^{-1 / \gamma}\right)\right|>\eta\right) \\
& =\mathbb{P}\left(\sqrt{\frac{n}{k}}\left|W_{n}\left(\left|\bar{H}^{(1)}\left(Z_{n-k: n} v\right)-p \frac{k}{n} v^{-1 / \gamma}\right|\right)\right|>\eta\right) \\
& \leq \mathbb{P}\left(\left|\frac{\bar{H}^{(1)}\left(Z_{n-k: n} v\right)}{v^{-1 / \gamma} k / n}-p\right|>\eta^{2} \frac{\epsilon^{2}}{4 v^{1 / \gamma}}\right)+\mathbb{P}\left(\sup _{0 \leq t \leq \frac{\epsilon^{2}}{4} \frac{k}{n}}\left|W_{n}(t)\right|>\eta \sqrt{k / n}\right) .
\end{aligned}
$$

It is clear that the first term, in the right-hand side of the inequality above, tends to zero as $n \rightarrow \infty$. On the other hand, since $\left\{W_{n}(t) ; 0 \leq t \leq 1\right\}$ is a martingale, then by using the classical Doob inequality, we have, for any $u>0$ and $T>0$,

$$
\mathbb{P}\left(\sup _{0 \leq t \leq T}\left|W_{n}(t)\right|>u\right) \leq \frac{\mathbf{E}\left|W_{n}(T)\right|}{u} \leq \frac{\sqrt{T}}{u} .
$$

Letting $T=\eta^{2} \frac{\epsilon^{2}}{4} \frac{k}{n}$ and $u=\eta \sqrt{k / n}$, yields that

$$
\mathbb{P}\left(\sup _{0 \leq t \leq \eta^{2} \frac{\epsilon^{2}}{4} \frac{k}{n}}\left|W_{n}(t)\right|>\eta \sqrt{k / n}\right) \leq \epsilon / 2 .
$$

This completes the proof of assertion $(i)$. To prove $(i i i)$, let us first show that

$$
\sqrt{\frac{n}{k}} \int_{h}^{Z_{n-k: n}} v^{-1} \mathbf{B}_{n}^{*}(v) d v=o_{\mathbb{P}}(1)
$$

For fixed $0<\eta, \epsilon<1$, we have

$$
\begin{aligned}
& \mathbb{P}\left(\left|\sqrt{\frac{n}{k}} \int_{h}^{Z_{n-k: n}} v^{-1} \mathbf{B}_{n}^{*}(v) d v\right|>\eta\right) \\
& \leq \mathbb{P}\left(\left|\frac{Z_{n-k: n}}{h}-1\right|>\epsilon\right)+\mathbb{P}\left(\sqrt{\frac{n}{k}} \int_{h}^{(1+\epsilon) h} v^{-1}\left|\mathbf{B}_{n}^{*}(v)\right| d v>\eta\right) .
\end{aligned}
$$

Since $Z_{n-k: n}=\left(1+o_{\mathbb{P}}(1)\right) h$, then the first term of the previous expression tends to zero as $n \rightarrow \infty$. On the other hand, it is easy to verify that $\mathbf{E}\left[\mathbf{B}_{n}^{*}(u) \mathbf{B}_{n}^{*}(v)\right]=$ $\min (\bar{H}(u), \bar{H}(v))-\bar{H}(u) \bar{H}(v)$, therefore $\mathbf{E}\left|\mathbf{B}_{n}^{*}(v)\right| \leq \sqrt{\bar{H}(v)}$. It follows that

$$
\mathbf{E}\left|\sqrt{\frac{n}{k}} \int_{h}^{(1+\epsilon) h} v^{-1} \mathbf{B}_{n}^{*}(v) d v\right| \leq \sqrt{\frac{n}{k}} \int_{h}^{(1+\epsilon) h} v^{-1} \sqrt{\bar{H}(v)} d v
$$

which, by a change of variables, is equal to $\int_{1}^{1+\epsilon} v^{-1} \sqrt{\frac{n}{k} \bar{H}(h v)} d v$. Since $\bar{H}(h v)$ is asymptotically equivalent to $v^{-1 / \gamma} k / n$ (uniformly in $v \geq 1$ ), then the latter integral 
is $\sim 2 \gamma\left(1-(1+\epsilon)^{-1 / 2 \gamma}\right)$ which tends to zero as $\epsilon \downarrow 0$. Consequently, (4.38) is true and we have

$$
\sqrt{\frac{n}{k}} \int_{1}^{\infty} v^{-1} \mathbf{B}_{n}^{*}\left(Z_{n-k: n} v\right) d v=\sqrt{\frac{n}{k}} \int_{1}^{\infty} v^{-1} \mathbf{B}_{n}^{*}(h v) d v+o_{\mathbb{P}}(1) .
$$

Then to have $(i i i)$, we need to show that

$$
\sqrt{\frac{n}{k}} \int_{1}^{\infty} v^{-1}\left(\mathbf{B}_{n}^{*}(h v)-\mathbb{B}_{n}^{*}\left(\frac{k}{n} v^{-1 / \gamma}\right)\right) d v=o_{\mathbb{P}}(1) .
$$

Observe that

$$
\begin{aligned}
& \sqrt{\frac{n}{k}} \int_{1}^{\infty} v^{-1}\left(\mathbf{B}_{n}^{*}(h v)-\mathbb{B}_{n}^{*}\left(\frac{k}{n} v^{-1 / \gamma}\right)\right) d v \\
& =\sqrt{\frac{n}{k}} \int_{1}^{\infty} v^{-1}\left(B_{n}\left(\theta-p \frac{k}{n} v^{-1 / \gamma}\right)-B_{n}\left(\theta-\bar{H}^{(1)}(h v)\right)\right) d v \\
& +\sqrt{\frac{n}{k}} \int_{1}^{\infty} v^{-1}\left(B_{n}\left(1-q \frac{k}{n} v^{-1 / \gamma}\right)-B_{n}\left(1-\bar{H}^{(0)}(h v)\right)\right) d v=: I_{n 1}+I_{n 2} .
\end{aligned}
$$

We only show that $I_{n 1}=o_{\mathbb{P}}(1)$, as similar arguments lead to the same result for $I_{n 2}$ (due to the symmetry structure of $I_{n 1}$ and $I_{n 2}$ ). Making use of the representation (4.37), we write

$$
\begin{gathered}
I_{n 1} \stackrel{d}{=} \sqrt{\frac{n}{k}} \int_{1}^{\infty} v^{-1}\left(W_{n}\left(\theta-p \frac{k}{n} v^{-1 / \gamma}\right)-W_{n}\left(\theta-\bar{H}^{(1)}(h v)\right)\right) d v \\
+W_{n}(1) \sqrt{\frac{n}{k}} \int_{1}^{\infty} v^{-1}\left(p \frac{k}{n} v^{-1 / \gamma}-\bar{H}^{(1)}(h v)\right) d v
\end{gathered}
$$

The increment $\left\{W_{n}\left(\theta-p \frac{k}{n} v^{-1 / \gamma}\right)-W_{n}\left(\theta-\bar{H}^{(1)}(h v)\right)\right\}$ of the Wiener process $W_{n}$ is $\mathcal{N}\left(0, \bar{H}^{(1)}(h v)-p \frac{k}{n} v^{-1 / \gamma}\right)$, then the absolute value of the first term of the right-hand side has an expectation which is less than or equal to

$$
\sqrt{\frac{n}{k}} \int_{1}^{\infty} v^{-1} \sqrt{\bar{H}^{(1)}(h v)-p \frac{k}{n} v^{-1 / \gamma}} d v .
$$

Likewise, the absolute value of the second term of right-hand side has an expectation which is less than or equal to

$$
\sqrt{\frac{n}{k}} \int_{1}^{\infty} v^{-1}\left|\bar{H}^{(1)}(h v)-p \frac{k}{n} v^{-1 / \gamma}\right| d v
$$

Routine manipulations, using assertion (i) in Lemma 4.1, on the last two quantities complete the proof. 
Proof of $\left[\mathcal{H}_{2}\right]-\left[\mathcal{H}_{3}\right] \Longrightarrow \sqrt{k}\left\{\frac{n}{k} \bar{H}^{(1)}(h)-p\right\} \rightarrow \mathbf{d}_{2}$

First, we show that

$$
\frac{1}{\sqrt{k}} \sum_{i=1}^{k}\left[\mathbf{p}\left(H^{\leftarrow}\left(1-\frac{i}{n}\right)\right)-p\right]=\sqrt{k}\left\{\frac{n}{k} \bar{H}^{(1)}(h)-p\right\}+O\left(\sqrt{k} \widetilde{\omega}_{n, k}\right),
$$

where $\widetilde{\omega}_{n, k}$ is a sequence such that $\sqrt{k} \widetilde{\omega}_{n, k} \rightarrow 0$, as $n \rightarrow \infty$. For convenience, we set $\varphi(s):=\mathbf{p}\left(H^{\leftarrow}(1-s)\right), 0<s \leq 1$. From the classical Riemann approximation, we have

$$
\begin{aligned}
& \left|\frac{1}{k} \sum_{i=1}^{k} \varphi\left(u+i \frac{v-u}{k}\right)-\frac{1}{v-u} \int_{u}^{v} \varphi(s) d s\right| \\
& \leq \sup _{\{u<x, y \leq v,|y-x| \leq(v-u) / k\}}|\varphi(y)-\varphi(x)|, \text { for } u<v<\infty .
\end{aligned}
$$

By letting $u=0$ and $v=k / n$, we get

$$
\left|\frac{1}{k} \sum_{i=1}^{k} \varphi\left(\frac{i}{n}\right)-\frac{n}{k} \int_{0}^{k / n} \varphi(s) d s\right| \leq \widetilde{\omega}_{n, k},
$$

where $\widetilde{\omega}_{n, k}:=\sup _{\{0<x, y \leq k / n,|y-x| \leq 1 / n\}}|\varphi(y)-\varphi(x)|$. That is, we have

$$
\frac{1}{k} \sum_{i=1}^{k} \mathbf{p}\left(H^{\leftarrow}\left(1-\frac{i}{n}\right)\right)=\frac{n}{k} \int_{0}^{k / n} \mathbf{p}\left(H^{\leftarrow}(1-x)\right) d x+O\left(\widetilde{\omega}_{n, k}\right) .
$$

Recall (1.4) and observe that $\mathbf{p}(z)=\left(\bar{H}^{(1)}\right)^{\prime}(z) / \bar{H}^{\prime}(z)$. Then we have

$$
\int_{0}^{k / n} \mathbf{p}\left(H^{\leftarrow}(1-x)\right) d x=\int_{0}^{k / n} \frac{\left(H^{(1)}\right)^{\prime}\left(H^{\leftarrow}(1-x)\right)}{H^{\prime}\left(H^{\leftarrow}(1-x)\right)} d x,
$$

which, by the change of variables $z=H^{\leftarrow}(1-x)$, equals $\int_{h}^{\infty}\left(H^{(1)}\right)^{\prime}(z) d z=$ $\bar{H}^{(1)}(h)$. Thus (4.40) readily follows. Next, we use the limit $\sqrt{k} \omega_{n, k}(C) \rightarrow 0$ of assumption $\left[\mathcal{H}_{3}\right]$. Observe that, for $n$ sufficiently large and all $C>0$, we have $1 / n<C \sqrt{k} / n$, then it is easy to verify that $\widetilde{\omega}_{n, k}<\omega_{n, k}(C)$. Hence $\sqrt{k} \widetilde{\omega}_{n, k} \rightarrow 0$, which yields that the second term of the right-hand side of the previous equation tends to zero as well. Finally, we use assumption $\left[\mathcal{H}_{2}\right]$ to conclude.

\section{REFERENCES}

Beirlant, J., Goegebeur, Y., Segers, J. \& Teugels, J. (2004). Statistics of ExtremesTheory and applications. Wiley.

Beirlant, J., Guillou, A., Dierckx, G. \& Fils-Villetard, A. (2007). Estimation of the extreme value index and extreme quantiles under random censoring. Extremes 10, $151-174$. 
Bingham, N.H., Goldie, C.M. \& Teugels, J.L. (1987). Regular Variation. Cambridge University Press.

Brahimi, B., Meraghni, D., Necir, A. \& Yahia, D. (2013). A bias-reduced estimator for the mean of a heavy-tailed distribution with an infinite second moment. $J$. Statist. Plann. Inference 143, 1064-1081.

Csörgö, M., Csörgő, S., Horváth, L. \& Mason, D.M. (1986). Weighted empirical and quantile processes. Ann. Probab. 14, 31-85.

Csörgő, S. (1996). Universal Gaussian approximations under random censorship. Ann. Statist. 24, 2744-2778.

Deheuvels, P. \& Einmahl, J.H.J. (1996). On the strong limiting behavior of local functionals of empirical processes based upon censored data. Ann. Probab. 24, $504-525$.

Einmahl, J.H.J., Fils-Villetard, A. \& Guillou, A. (2008). Statistics of extremes under random censoring. Bernoulli 14, 207-227.

Einmahl, J.H.J. \& Koning, A.J. (1992). Limit theorems for a general weighted process under random censoring. Canad. J. Statist. 20, 77-89.

Fraga Alves, I., Gomes, I. \& de Haan, L. (2007). A note on second order condition in extreme value theory: linking general and eavy tail conditions. Revstat 5, 285-304.

Gomes, M.I. \& Neves, M.M. (2011). Estimation of the extreme value index for randomly censored data. Biometrical Letters 48, 1-22.

de Haan, L. \& Stadtmüller, U. (1996). Generalized regular variation of second order. J. Australian Math. Soc. (Series A) 61, 381-395.

de Haan, L. \& Ferreira, A. (2006). Extreme Value Theory: An Introduction. Springer.

Hill, B.M. (1975). A simple general approach to inference about the tail of a distribution. Ann. Statist. 3, 1163-1174.

Koning, A.J. \& Peng, L. (2008). Goodness-of-fit tests for a heavy tailed distribution. J. Statist. Plann. Inference 138, 3960-3981.

Reiss, R.D. \& Thomas, M. (1997). Statistical Analysis of Extreme Values with Applications to Insurance, Finance, Hydrology and Other Fields. Birkhäuser.

Worms, J. \& Worms, R. (2014). New estimators of the extreme value index under random right censoring, for heavy-tailed distributions. Extremes 17, no. 2, 337358. 